\title{
Transition from squeezing to dripping in a microfluidic T-shaped junction
}

\author{
M. DE MENECH ${ }^{1}$, P. GARSTECKI I , F. JOUSSE \\ AND H. A. STONE \\ ${ }^{1}$ Max-Planck Institute for the Physics of Complex Systems, Nöthnitzer Str. 38, \\ 01187, Dresden, Germany \\ ${ }^{2}$ Institute of Physical Chemistry, Polish Academy of Sciences, Kasprzaka 44/52, \\ 01-224, Warsaw, Poland \\ ${ }^{3}$ Unilever Corporate Research, Colworth House, Sharnbrook, Bedfordshire, MK44 1LQ, UK \\ ${ }^{4}$ School of Engineering and Applied Sciences, Harvard University, Cambridge, MA 02138, USA
}

(Received 12 May 2006 and in revised form 5 September 2007)

We describe the results of a numerical investigation of the dynamics of breakup of streams of immiscible fluids in the confined geometry of a microfluidic T-junction. We identify three distinct regimes of formation of droplets: squeezing, dripping and jetting, providing a unifying picture of emulsification processes typical for microfluidic systems. The squeezing mechanism of breakup is particular to microfluidic systems, since the physical confinement of the fluids has pronounced effects on the interfacial dynamics. In this regime, the breakup process is driven chiefly by the buildup of pressure upstream of an emerging droplet and both the dynamics of breakup and the scaling of the sizes of droplets are influenced only very weakly by the value of the capillary number. The dripping regime, while apparently homologous to the unbounded case, is also significantly influenced by the constrained geometry; these effects modify the scaling law for the size of the droplets derived from the balance of interfacial and viscous stresses. Finally, the jetting regime sets in only at very high flow rates, or with low interfacial tension, i.e. higher values of the capillary number, similar to the unbounded case.

\section{Introduction}

Because of the small size of the microchannels (widths of the order of 10 to $100 \mu \mathrm{m}$ ) and typical flow rates $\left(1 \mu 1 \mathrm{~s}^{-1}\right)$, flows in microfluidic systems are generally dominated by viscous effects. Two characteristics of microflows, i.e. the laminar flow and the typically large values of the Péclet number (measuring the ratio of the convective to diffusive transport), allow for an extensive control both in space and time over the transport of chemical substances (Kenis, Ismagilov \& Whitesides 1999; Stone, Strook \& Ajdari 2004; Squires \& Quake 2005). This control, in conjunction with the ease of fabrication of the microfluidic devices (Duffy et al. 1998; McDonald et al. 2000), is one of the main features driving the interest in microfluidic systems for engineering and research applications.

The laminar flow of a single Newtonian fluid at low Reynolds numbers, which is described by linear equations and boundary conditions, is to be contrasted with a wide class of nonlinear phenomena which have been uncovered with the first experiments on two-phase flows in microfluidic systems (Thorsen et al. 2001; Ganán-Calvo \& 


\section{Application}

Chemical processing

Micromixing inside drops

Drops and slugs as mixing elements

High-throughput screening

Kinetic analyses

Organic chemistry

Bioanalysis

Diagnostic assays

Handling and/or analysis of living cells

Molecular evolution

Material science

Anisotropic particles

Microcapsules

Colloidal shells

Review articles
Reference

Song et al. (2003)

Gunther et al. (2004); Garstecki et al. (2005a)

Zheng et al. (2003); Zheng \& Ismagilov (2005)

Song \& Ismagilov (2003)

Cygan et al. (2005)

Sia et al. (2004)

He et al. (2005)

Cornish (2006)

Jeong et al. (2004, 2005); Nisisako et al. (2004);

Dendukuri et al. (2005); Xu et al. (2005)

Takeuchi et al. (2005)

Subramaniam et al. (2005)

Gunther \& Jensen (2006); Song et al. (2006)

TABLE 1. Examples of applications of multiphase flow in microfluidic devices.

Gordillo 2001; Anna, Bontoux \& Stone 2003; Dreyfus, Tabeling \& Willaime 2003). The existence of an interface and the influence of interfacial tension introduce strong nonlinearities in the flow, which are responsible for the appearance of a range of novel effects, some of which are particular to microfluidic systems when the interfacial dynamics is strongly influenced by the confinement of the fluids by the walls of the channel (Garstecki et al. 2005b,c,d, 2006; Guillot \& Colin 2005).

The interest in detailed understanding of the emulsification processes in microfluidic systems is additionally motivated by the wide range of work on applications of microfluidic multiphase flows (see table 1). For example, microfluidics offers new routes and better control to chemistry, including chemistry inside small containers (e.g. Li, Zheng \& Harris 2000; Song, Chen \& Ismagilov 2006), control of dispersion (e.g. Pedersen \& Horvath 1981), etc., approaches that have a long history in chemical engineering. Most of the applications require precise control over the process of formation of droplets or bubbles (e.g. Basaran 2002), and characterization or, preferably, understanding of the scaling laws that describe the volume of the bubbles or droplets formed in the devices as a function of the material (e.g. viscosities, interfacial tension) and flow parameters (e.g. pressures or rates-of-flow applied to the system). Understanding the flows is closely related to more classical studies of drop breakup and emulsification in sheared unbounded fluid systems (Rallison 1984; Stone 1994). Nevertheless, as we shall discuss, the confinement that naturally accompanies flow in small devices has significant qualitative and quantitative effects on the drop dynamics and breakup (Garstecki et al. 2005d, 2006; Guillot \& Colin 2005).

Several methods of formation of both bubbles and droplets have already been described. Table 2 provides references to several recent experimental reports. Numerical simulations of breakup in microfluidic geometries have also been conducted, see for example studies of axisymmetric geometries by Jensen, Stone \& Bruus (2006), Suryo \& Basaran (2006) and Zhou, Yue \& Feng (2006). For a thorough up-to-date review of drop formation in microfluidic devices see Christopher \& Anna (2007). Here we report fully three-dimensional numerical simulations to understand and characterize drop formation in a microfluidic T-junction geometry (figure 1) which was first introduced for the controlled formation of water-in-oil dispersions by 
Subject

Planar geometries

Review article

Planar geometries

Flow-focusing

Crossflow

Diverging flow T-junction Axisymmetric geometries

Nonplanar geometries
Reference

Christopher \& Anna (2007)

Anna et al. (2003); Dreyfus et al. (2003); Cubaud \& Ho (2004); Garstecki et al. (2004); Xu \& Nakajima (2004);

Ward et al. (2005)

Blackmore et al. (2001); Thorsen et al. (2001); Song \& Ismagilov (2003); Okushima et al. (2004); Zheng et al. (2003); Gerdts et al. (2004); Tice et al. (2004); Dendukuri et al. (2005);

Guillot \& Colin (2005); Garstecki et al. (2006)

Link et al. (2004); Engl et al. (2005)

Ganán-Calvo \& Gordillo (2001); Jeong et al. (2004, 2005);

Takeuchi et al. (2005); Utada et al. (2005)

Sugiura et al. $(2001,2005)$

TABLE 2. Examples of droplet and bubble formation in microfluidic devices. Given the rapid growth in the number of such two-phase-flow studies, the above references are representative of the kinds of studies that have been performed.

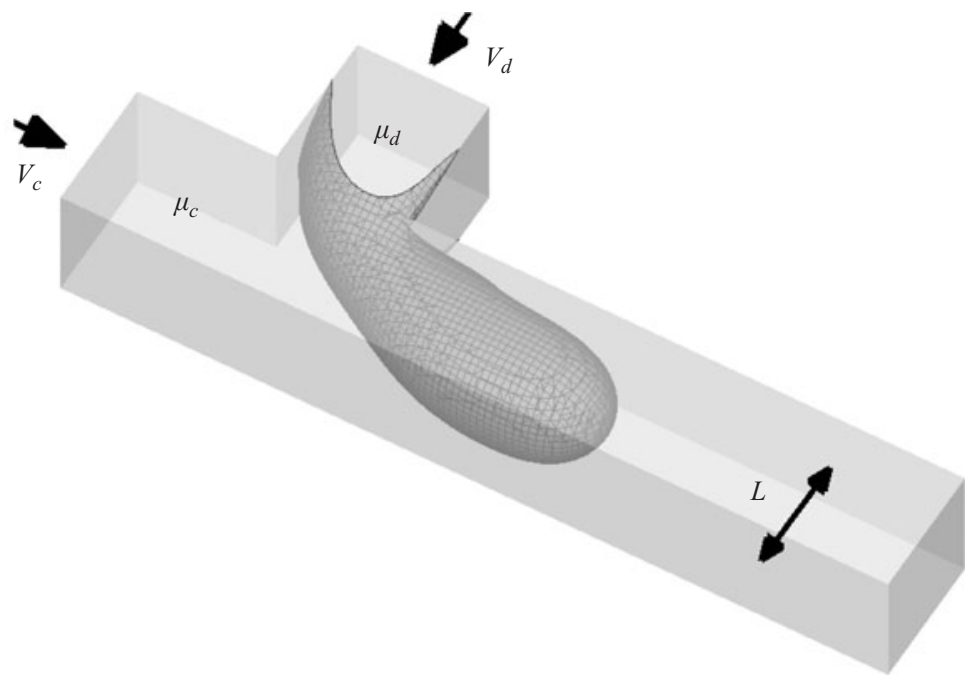

FIGURE 1. Diagram of a T-junction with crossflow. The channels have square cross-section with side $L . v_{c}$ and $v_{d}$ are the mean flow velocities of the continuous and dispersed phases, while $\mu_{c}$ and $\mu_{d}$ are the corresponding shear viscosities.

Thorsen et al. (2001). The authors made the reasonable suggestion that the dynamics of droplet formation is dominated by the balance of tangential shear stresses and interfacial tension (i.e. the capillary number) as expected in unbounded shear flows, via an analogy to breakup processes in shear and extensional flows (Taylor 1934; Rallison 1984; Stone 1994). A detailed experimental study of the T-junction configuration (Garstecki et al. 2006) identified a different (squeezing) mechanism that is directly connected to the confined geometry in which the drop is formed (see also Guillot \& Colin 2005). It was proposed that when the capillary number is sufficiently small, the dominant contribution to the dynamics of breakup arises from the buildup of pressure upstream of the emerging droplet (Garstecki et al. 2005d, 2006). This model 
results in a scaling law for the size of the droplets that is independent of the value of the capillary number and includes only the ratio of the rates of flow of the two immiscible fluids.

The numerical results that we present here provide a unifying picture of the dynamics of formation of droplets in microfluidic $\mathrm{T}$-junction geometries that includes both of the aforementioned squeezing (Garstecki et al. 2005d, 2006) and shear-driven (Thorsen et al. 2001) types of breakup; for another three-dimensional simulation of drop formation at a T-junction, see van der Graaf et al. (2006). We confirm the existence of the 'rate-of-flow controlled' or 'squeezing' breakup mechanism at low values of the capillary number $C a$. We provide the details of the dynamics, which includes fluctuations of pressure upstream of the immiscible tip postulated by Garstecki et al. (2006). We identify a critical value of the capillary number at which the system transits into a shear-dominated or dripping mechanism of droplet formation. Also, we indicate the differences in drop formation in confined and unbounded systems. Finally, similarly to breakup into an unbounded fluid, we observe a transition from dripping to jetting at larger values of the capillary number. We note that although jetting refers to the formation of long threads prior to formation of a drop, which is usually associated with inertial effects of the internal phase, here we have a case where shear in the external phase drives a jetting transition at low to modest Reynolds numbers (e.g. Utada et al. 2007).

In the following section, we define the geometry and the parameters of the system that we study and introduce the important dimensionless quantities. In $\S 3$, we describe the numerical methods employed in our work. In $\S 4$, we detail the results, both for the low- and high-capillary-number regimes, and we summarize our observations in $\S 5$.

\section{Description of the system}

For a planar geometry, the characteristic dimensions of the T-shaped junction are the height $h$, and the widths of the main and side channels. We will consider the simplest case in which the widths of both ducts equal $L$, and the channels have a square cross-section $h=L$ (figure 1). The dispersed phase is injected into the main channel from the side inlet. For simplicity, we set the densities of both phases equal; we expect that this choice has negligible influence on the results, since in most microfluidic configurations buoyancy-driven speeds are much smaller than the actual flow speeds. Besides the width $L$, which is constant, the problem is fully described by six parameters characterizing the flow and material properties of the fluids. These parameters are the mean speeds of the continuous and dispersed phases, $v_{c}$ and $v_{d}$ respectively, the viscosities of the two fluids $\mu_{c}$ and $\mu_{d}$, the interfacial tension $\gamma$, and the density $\rho$. We will assume perfect wetting for the continuous phase, while the dispersed fluid does not wet the walls. The rescaled volume $V=V_{d} / L^{3}$ of the droplets formed in the device is the eighth physical quantity, and following the Buckingham- $\Pi$ theorem, it can be described as a function of four dimensionless parameters. We chose the following groups: the capillary number calculated for the continuous phase, $C a=\mu_{c} v_{c} / \gamma$, the Reynolds number $R e=\rho v_{c} L / \mu_{c}$, the viscosity ratio $\lambda=\mu_{d} / \mu_{c}$, and the flow rate ratio $Q=v_{d} / v_{c}=Q_{d} / Q_{c}$, where $Q_{d}=v_{d} L^{2}$ and $Q_{c}=v_{c} L^{2}$ are the flow rates at the two inlets. The pressure will be rescaled by the typical viscous shear stress $\mu_{c} v_{c} / L$, while the time unit is $L / v_{c}$.

For the flow regimes under consideration, the Reynolds number is small $(\operatorname{Re}<1)$, and does not influence the droplet size, which leaves us with the three governing parameters: $C a, \lambda$ and $Q$. Our main focus is the discussion of the transition 
from the interfacial-tension dominated to the shear-dominated regime, which is best characterized by considering the effects of the capillary number on the droplet size. Within this framework, we will also consider the influence of $\lambda$ and $Q$.

\section{The numerical model}

We use a phase-field model (De Menech 2006) to simulate numerically the flow of the two immiscible fluids at the T-junction. In common experimental configurations, low capillary numbers characterize the microfluidic flows, hence surface tension stresses are large in comparison to viscous stresses. These flows can be effectively tackled numerically with the diffuse interface method that we employ here, which models the phase boundary separating the two fluids as a diffuse region. The equilibrium properties of the mixture, including phase behaviour, wetting properties and the concentration profile in the interface region, are derived from a generalized free-energy functional, which also determines the diffusive and capillary forces in the transport equations. The transport equations are solved on a three-dimensional grid, and the method has been tested successfully in the case of droplet breakup in a microfluidic T-junction with diverging flow (De Menech 2006).

\subsection{Modelling multiphase flow}

The key issue in the numerical modelling of droplet formation and breakup in microfluidic devices is the requirement of a consistent and robust description of the effects related to the large interfacial tensions, which dominate over inertial and viscous stresses. As for the broader context of multiphase flow simulation, we can choose from the two main approaches: interface tracking and interface capturing methods (for an introductory review focused on microfluidics, see for example Cristini \& Tan 2004).

In interface tracking methods, the displacement of the boundary surface is followed explicitly by an adapting mesh, which represents the discrete approximation of the classical sharp interface limit. The main advantage of this approach is the accurate description of the interface, which comes at the expense of a greater complexity of the algorithms that are required to manage the motion and addition of mesh nodes. The handling of the singularities associated with surface merging and breakup represents a difficult technical problem, since the remeshing procedure has to cope with the changes in topology (see, e.g. Cristini, Balwzdziewicz \& Loewenberg 1998; Wilkes, Phillips \& Basaran 1999). In this framework, a few examples are available for the description of the dynamics of droplets pinned at or sliding on flat surfaces, both in two- and three-dimensional studies (Feng \& Basaran 1994; Li \& Pozrikidis 1996; Schleizer \& Bonnecaze 1999).

In interface capturing methods, the computational mesh remains fixed and the boundary discontinuities are smeared out over the finite width $\xi$ of a diffuse interface, whose location is reconstructed from the gradients of an auxiliary scalar field. Surface stresses are included in the Navier-Stokes equation as volume forces that depend on the spatial derivatives of the auxiliary scalar field, such that the momentum transport equation remains consistent with the single-phase equation in the bulk regions. The lower bound to the manageable interface thickness $\xi$ is determined by the mesh spacing, and the discontinuity of the sharp interface limit behaviour is recovered asymptotically as $\xi$ becomes negligible with respect to the characteristic length of the flow $L$. Broadly speaking, we can distinguish two mainstream approaches in the class of interface capturing methods, based on the physical interpretation of the auxiliary 
scalar field. In phase-field models (also called diffuse interface models; see Anderson, McFadden \& Wheeler 1998), the behaviour of the scalar field in the transition region strictly follows from a variational principle applied to a generalized free-energy functional, which determines the equilibrium and non-equilibrium properties of the system. On the other hand, in the case of popular techniques such as the volume of fluid (VOF) method (Scardovelli \& Zaleski 1999), or the level-set method (Osher \& Fedkiw 2001), the scalar field (also known as a colour function in VOF methods) is merely an indicator of the different phases; its profile across the transition region has no meaning, other than serving to define the position of the phase boundary.

Phase-field methods were developed to investigate phase transition phenomena, such as nucleation, evaporation and coarsening (Cahn 1965), where the diffuse interface provides a mean field description of the concentration or density profile. The phasefield version of the Navier-Stokes equations converges to the classical sharp interface behaviour as the interface thickness is reduced to zero along with the diffusivity (Jacqmin 1999). In the same manner, contact line dynamics can be related to that of immiscible fluids (Jacqmin 2000).

In this paper, we will be using the phase-field model in which the capillary stresses at the interfaces are represented as volume forces, and are distributed over the characteristic thickness $\xi$ of the diffuse phase boundary. This important feature of the model allows the treatment of multiphase flows with relatively coarse grids, since typically only a few mesh points are required in order to resolve the smooth variation of the order parameter across the interface. The interface thickness should be compared to a characteristic length of the system, which could be the radius of a droplet or the size of the domain (here $L$ ) in the case of confined flow, leading to the definition of the Cahn number $C=\xi / L$. The droplet dynamics of two immiscible fluids, described classically in the sharp interface limit $\xi \rightarrow 0$, is exactly recovered as $C$ and the diffusivity goes to zero (Jacqmin 1999).

There are two possible ways to interpret the results of the phase-field model. The first one is to relate $\xi$ to the physical width of the interface for real fluids, which is of the order of $1 \mathrm{~nm}$ or larger. In this approach, our simulations should, within the continuum model, reflect the actual flow of fluids at the nano-scale, with typical channel widths of the order of $10 \mathrm{~nm}$. Another interpretation is to consider the diffuse interface model as an approximation of the flow of immiscible fluids at the microscale, which is justified when the interface thickness $\xi$ is small compared with the characteristic length of the flow $L$. Despite $\xi$ not being the interface thickness separating real bulk fluids (e.g. since we typically choose $C=1 / 20$ for numerical convenience, then $\xi \sim 5 \mu \mathrm{m}$ when simulating drops in $100 \mu \mathrm{m}$ channels), the experimentally observed droplet dynamics in microfluidic devices can be reproduced effectively (De Menech 2006).

De Menech (2006) showed that the numerical model, despite the limitations related to the coarse description of the interface description, is capable of capturing the quantitative details of the dynamics of droplet breakup in a T-junction, in agreement with the experimental results for water droplets in a continuous oil phase. The results that we describe in this report, which are expressed in terms of the non-dimensional groups, also match closely the experimental observation of flow of droplets and bubble formation in microfluidic T-junctions (Garstecki et al. 2006).

\subsection{The phase-field model}

Phase-field models and a range of related numerical methods based on the generalized free-energy functional approach (lattice Boltzmann) have already been applied to 
the modelling of droplet dynamics in unbounded flow or in the presence of solid boundaries, including droplet deformation and breakup (Wagner \& Yeomans 1997; Badalassi, Ceniceros \& Banerjee 2003; Wagner, Wilson \& Cates 2003; Kim 2005), droplet formation (Kuksenok et al. 2003), spreading on patterned surfaces (Dupuis \& Yeomans 2004), and droplet formation in axially symmetric flow-focusing devices (Zhou et al. 2006). In this section, we summarize the main features of the phase-field model we used. A more complete description is given in De Menech (2006).

We consider a mixture of two fluids, $A$ and $B$, with the Cahn-Hilliard-van der Waals form for the free energy

$$
F[n, \varphi]=\int \mathrm{d} \boldsymbol{r}\left\{\frac{\kappa n}{2}|\nabla \varphi|^{2}+n W(\varphi)+f(n)\right\},
$$

where $n=n_{A}+n_{B}$ is the total particle number density, $\varphi=n_{B} / n$ is the molar fraction of component $B, \kappa$ determines the interface thickness, $\xi \propto \sqrt{\kappa}$, and the interfacial tension, $\gamma \propto \kappa / \xi$ (De Menech 2006). In (3.1), $f$ is the sum of the free-energy densities of the pure components, which for simplicity is assumed to depend only on the total density. Also, $W$ is the free energy of mixing, having the characteristic double-well shape below the critical temperature, which therefore determines the coexistence of the $A$-rich and $B$-rich separate phases, with corresponding concentrations $\varphi_{A}$ and $\varphi_{B}$.

We will consider the incompressible limit, $n=$ const, such that the equilibrium concentration satisfies the Euler-Lagrange equation

$$
\mu_{c h}(\boldsymbol{r})=-\kappa \nabla^{2} \varphi(\boldsymbol{r})+W^{\prime}(\varphi(\boldsymbol{r}))=\mu_{\text {coex }},
$$

where $\mu_{c h}(\boldsymbol{r})=\delta F / \delta \varphi(\boldsymbol{r})$ is the chemical potential difference, and $\mu_{c o e x}$ is the Lagrange multiplier which fixes the total molar fraction. In the framework of the Cahn-Hilliard theory of diffuse interfaces, the interaction of the fluid components with a wall is introduced by adding to the functional (3.1) a surface energy term (Cahn 1977), which fixes the relative affinity of the two components, $A$ or $B$, for the solid boundaries. The surface energy therefore determines the wetting properties of the two phases, which can be expressed in terms of the equilibrium wetting angle. We choose the surface free energy such that the $A$ component wets the walls, while the $B$ component is repelled.

In non-equilibrium conditions, local imbalances of the chemical potential $\mu_{c h}(\boldsymbol{r})$ will generate diffusion currents which tend to restore the configuration satisfying (3.2). The advection-diffusion equation for the phase field is therefore

$$
\frac{\partial \varphi}{\partial t}=-\nabla \cdot(\varphi \boldsymbol{v})+\Lambda \nabla^{2}\left[-\kappa \nabla^{2} \varphi+W^{\prime}(\varphi)\right]
$$

where $\Lambda$ is the mobility coefficient. Since the flow is considered incompressible, $\nabla \cdot v=0$. The expression for the pressure tensor, which includes the capillary forces generated by the presence of the interface, follows directly from the free-energy functional (3.1), based on the Gibbs-Duhem equation or on Noethers theorem (Anderson et al. 1998; De Menech 2006). The fluid momentum equation, besides the nonlinear advection term, contains both reactive and dissipative forces, depending, respectively, on the pressure tensor and the shear viscosity $\mu$, which depends on $\varphi$. The Navier-Stokes equation is

$$
\rho \frac{\partial \boldsymbol{v}}{\partial t}=-\rho \nabla \cdot(\boldsymbol{v} \boldsymbol{v})-\nabla p+\nabla \cdot\left(\mu\left[\nabla \boldsymbol{v}+(\nabla \boldsymbol{v})^{T}\right]\right)+\frac{\gamma \xi}{\Omega} \nabla \cdot(\nabla \varphi \nabla \varphi),
$$


where the last term comes from the non-isotropic part of the pressure tensor, as first introduced by Korteweg, and $\Omega$ is a dimensionless number that depends on the choice of the mixing potential $W$ (De Menech 2006).

Finally, we remark that in the case of contact line dynamics, the results of a phasefield model match those of immiscible fluids in the far-field region, i.e. at a distance from the contact line which is larger than the characteristic interface thickness $\xi$ (Jacqmin 2000). Similarly to the case of droplet breakup and interface merging, the dynamics of diffuse interface models in the near-field region have the significant advantage of removing the singularities that cause the breakdown of the sharp interface limit description of these phenomena. In our three-dimensional simulations, it is not strictly possible to identify a far-field region, since the pipe diameter is comparable with the interface width for the values of the Cahn number we have used. In other words, the adoption of the phase-field model for the description of two immiscible fluids is not justified a priori. On the other hand, as for the case of droplet breakup in a T-junction (De Menech 2006), we will show that the agreement with the experiments corroborates the validity of our approach, which in essence aims at classifying the variety of patterns observed in multiphase flows in microfluidic devices.

\subsection{The numerical method}

The transport equations (3.3) and (3.4) are discretized on a uniform three-dimensional Cartesian grid with staggered velocities; the molar fraction and pressure fields are collocated at the centres of the cubic cells, while the velocity components are placed on the faces, and the boundaries of the simulated domain always coincide with a face of a grid cell, be it a wall, an inlet or a pressure outlet. The time evolution is implemented with a fully implicit Euler scheme, which is first order in time (see De Menech 2006). The boundary condition (BC) for the molar fraction $\varphi$ is of Neumann type at the outlet, $\boldsymbol{n} \cdot \nabla \varphi=0$, where $\boldsymbol{n}$ is the vector normal to the outlet. A Dirichlet BC is set at the inlets, $\varphi=\varphi_{A}$ at the main channel inlet and $\varphi=\varphi_{B}$ at the side inlet. The BCs for the fluid velocity $v$ are of Dirichlet and Neumann type at the inlets and outlet, respectively, with the inlet velocity profile set equal to the fully developed Poiseuille flow in a square pipe. The BCs for the pressure field $p$ are of Neumann and Dirichlet type at the inlet and outlet, respectively. Finally, on all boundaries it is assumed that the chemical potential gradient is zero, so that locally there are no contributions to a flux of mole fraction. With respect to the actual discretization the typical grid spacing $\Delta x / L$ was equal to $C$, which implies 20 grid points across the tube diameter. For the smallest domain constructed, with a tube length of $6 L$ and a side inlet of length $L$, we have therefore 56000 grid points in the three-dimensional domain.

Given the stress tensor $\boldsymbol{T}$ in the Navier-Stokes equation (3.4), which is the sum of the viscous stress tensor $\mu\left[\nabla v+(\nabla v)^{T}\right]$ and the negative of the pressure tensor, $\boldsymbol{P}=p \boldsymbol{I}+(\gamma \xi / \Omega) \nabla \varphi \nabla \varphi$, then at the outlet we have $T_{z z}=P_{z z}=p=p_{0}$ (where $z$ is normal to the boundary); at the outlet, the pressure is constant and equal to the isotropic part of the pressure tensor. This set of boundary conditions at the outlet is equivalent to the assumption that the flow is fully developed at the end of the tube, and that there is no net flux of the two components $A$ and $B$ (note that at the inlet there is still a net influx because of the Dirichlet BC).

The constant pressure condition and the condition $\boldsymbol{n} \cdot \nabla \varphi=0$ at the outlet deforms the shape of droplets which touch the outlet, since the Laplace pressure difference here is forced to be zero and the curved interface is rapidly stretched and stabilized to become perpendicular to the boundary. Very long jets which touch the outlet are, in fact, stabilized, with their shape remaining stationary. These effects cannot be avoided 
in a finite simulation domain. On the other hand, such effects play a role only when the droplet or the thread is very close to the boundary (a distance comparable to the interface thickness $\xi$ ), and the pressure fluctuations associated with these events have very high frequency, and do not affect the slow dynamics of droplet formation inside the junction. These two facts still enable us to trust reliably the breakup dynamics observed far from the outlet, as for the squeezing and dripping regimes. We also performed a few tests to check that the size of droplets formed in the channel is not affected by changing the length of the pipe downstream from the T-junction.

\section{Results}

Before we present our results, we sketch the characteristics of the two main dynamical models for breakup in a microfluidic $\mathrm{T}$-junction: shear-driven breakup (Thorsen et al. 2001), which is derived from the balance of shear and interfacial stresses, and the rate-of-flow-controlled breakup (Garstecki et al. 2005d, 2006), which was proposed for systems operating at low values of the capillary number and is based on the evolution of pressure in the continuous phase upstream of the tip of the dispersed phase.

In shear-driven breakup, the volume of the droplet can be estimated from a balance of the viscous drag that the continuous fluid exerts on the emerging droplet and the interfacial force that opposes the elongation of the neck, which connects the reservoir of the dispersed fluid with the droplet (e.g. Umbanhowar, Prasad \& Weitz 2000; Thorsen et al. 2001). This model leads to a relation in which the diameter of the droplet is inversely proportional to the capillary number calculated for the flow of the continuous liquid. Because the drag force exerted on the droplet depends only very weakly on the viscosity of the droplet, within the shear-driven regime, the viscosity of the dispersed phase does not influence the size of droplets appreciably (e.g. see discussion of flow past a drop in Batchelor 1967). This effect has been confirmed experimentally by Cramer, Fischer \& Windhab (2004).

Qualitatively, the rate-of-flow-controlled breakup (Garstecki et al. 2005d, 2006) can be described as follows (see figure 1): the tip of the stream of the immiscible fluid enters the main channel, and because interfacial stresses dominate the shear stresses, the tip blocks almost the entirety of the cross-section of the main channel; the shear stresses exerted on the tip by the continuous fluid are not strong enough to deform the tip significantly away from an area minimizing shape. As a result, the continuous phase fluid is confined to thin films between the tip of the other immiscible fluid and the walls of the device. Flow in these thin films is subject to an increased viscous resistance, which leads to a build-up of pressure in the continuous phase upstream of the tip (Stone 2005). This pressure is larger than the pressure in the immiscible tip, and the continuous fluid displaces the interface or squeezes the neck of the inner fluid, which leads to breakup and detachment of a droplet. Notably, within this regime the breakup is not driven by interfacial stresses, at least not until the very last stage; the speed at which the neck of the immiscible thread collapses is proportional to the rate of flow of the continuous fluid, and does not depend significantly on the value of the interfacial tension, or on the values of the viscosities of either of the two fluids (Garstecki et al. 2005d). Within this regime, we can expect that the volume of the droplet will be a function of the ratio of the rates of flow of the two fluids, and will not depend strongly on the value of the capillary number (Garstecki et al. 2006).

The qualitatively different predictions of each of the two models of breakup make it possible to distinguish between them by inspecting how the volume of the droplets 
depends on the material (viscosities and interfacial tension) and flow (rates of flow) parameters. In this case, an advantage of numerical simulations over the experiments is the control over the different parameters. In the experiments, for example, it is difficult to change the interfacial tension between the two fluids over a wide range of values without introducing dynamic interfacial tension effects (at low concentrations of surfactant), or changing the wetting properties of the two fluids. In contrast, in simulations, it is straightforward to change the value of any parameter without affecting any others. Here we present the results of three-dimensional simulations for a range of the values of $\mathrm{Ca}$ that is broad enough to observe all three mechanisms of breakup in the confined geometry of a T-junction: squeezing, dripping and jetting.

\subsection{Overview of the dynamics of the system}

As the dispersed phase enters from the side inlet into the main channel, it is distorted by the stresses exerted by the outer fluid, which act to squeeze and stretch the inner fluid in the downstream direction (see figure 1). Interfacial tension tends to resist this external force by pulling the thread upstream and the dynamics of droplet formation in the channel will depend on the relative strengths of viscous, pressure and surface stresses. After an initial transient, whose duration in time grows linearly with the capillary number and during which drops form aperiodically, we observe the regular formation of monodisperse droplets.

In figure 2, we show a sequence of images illustrating drop formation and report the measured dimensionless droplet volume, $\mathrm{V}$, as a function of $\mathrm{Ca}$ in two different scenarios: allowing the viscosity ratio to change, while keeping $Q$ fixed, or, conversely, varying $Q$ while fixing $\lambda$. We can clearly identify two different regions in both diagrams, at low and high $\mathrm{Ca}$, respectively. For a range of low $\mathrm{Ca}$, the droplet size does not change significantly, whereas beyond the critical value $C a_{c} \approx 0.015$, which is not a strong function of viscosity ratio, the droplet size decreases rapidly with the capillary number. Also, changing the viscosity contrast has little or no effect at low $\mathrm{Ca}$, whereas it modifies quite sensibly the droplet volume at high $\mathrm{Ca}$. The visualization of the droplet formation process (figure $2 a-c$ ) shows that the two regimes of drop formation have qualitatively different characteristics. At low $\mathrm{Ca}$, the incoming thread tends to occupy the full width of the main channel, with the breakup occurring at the junction (figure $2 a$ ). At high $\mathrm{Ca}$, the dispersed fluid occupies only part of the main channel, and smaller droplets are formed downstream of the T-junction (figure $2 b-d$ ), with dynamics that resembles more and more that of the classical pendant-drop problem in a coflow geometry (Umbanhowar et al. 2000; Cramer et al. 2004). We will show that this dripping regime has significant differences from the unbound coflow case, since, even for high $C a$, the droplets occupy a significant portion of the main channel, which in turn affects the flow field of the outer continuous-phase fluid. In addition, we report in figure $2(d)$ some snapshots of the development of a thread for a high capillary number: the droplet detachment point gradually moves downstream, until a stable jet is formed, whose length in this case is limited by the size of the simulation domain.

\subsection{Low $\mathrm{Ca}$ : the squeezing regime}

Numerical simulations allow access to all of the dynamical variables of the system. For example, we can monitor the evolution of the droplet formation by looking at the time dependence of the pressure in the continuous fluid immediately upstream of the tip of the dispersed phase. This evolution of pressure at the junction lies at the heart of the interfacial dynamics in the squeezing regime (Garstecki et al. 2005d, 2006). Qualitatively, at low values of $\mathrm{Ca}$, the tip of the dispersed fluid effectively 
(a)

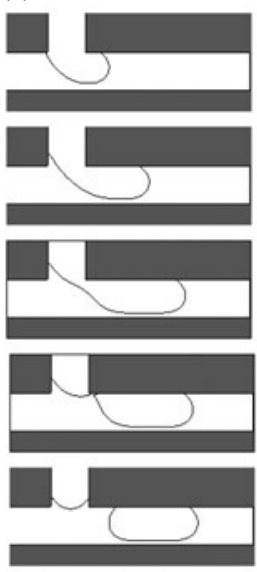

(e)

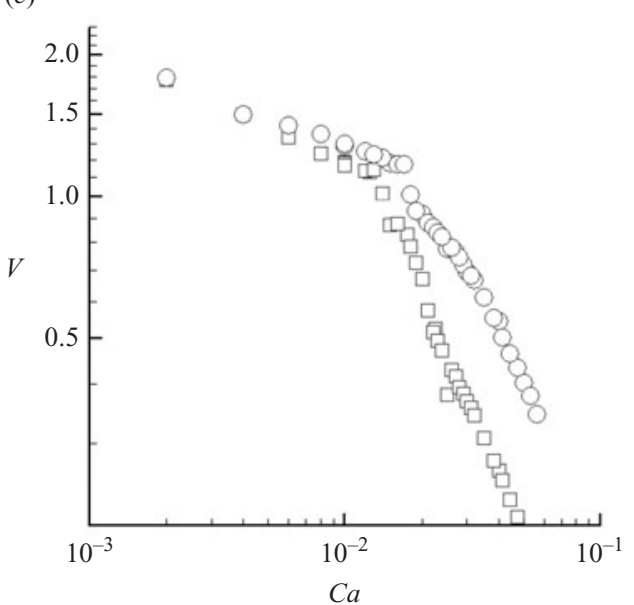

(c)
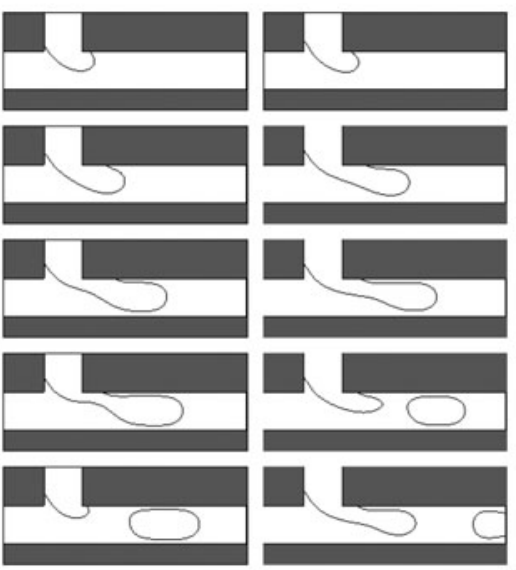

$(f)$ (d)

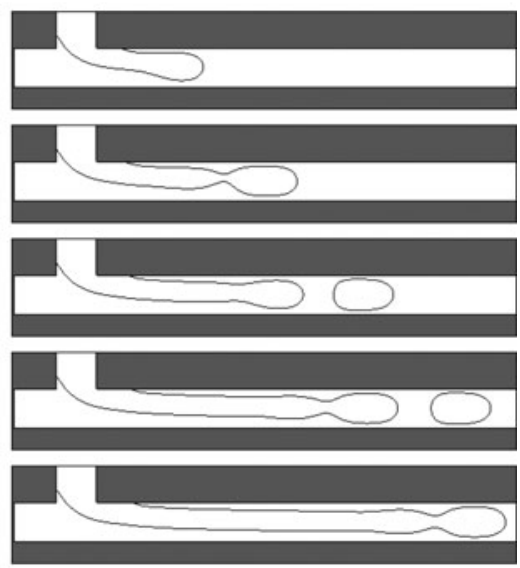

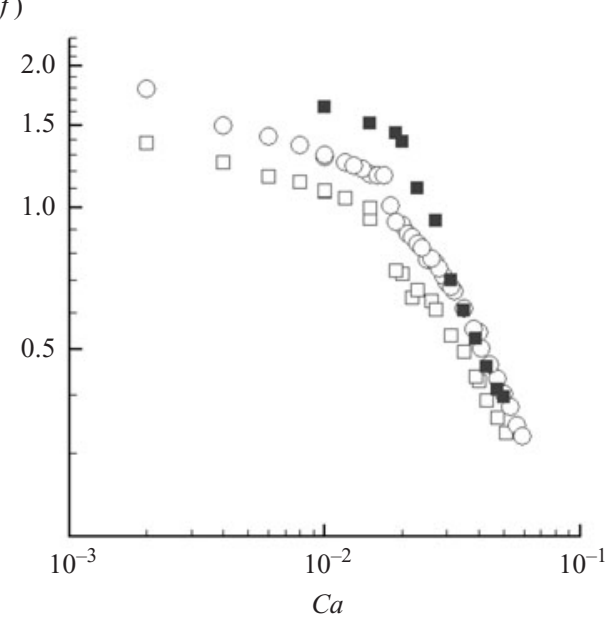

FIGURE 2. Drop formation as a function of capillary number. Two regimes of droplet formation are clearly distinguishable: at low $C a-(a) \quad C a=0.004, \lambda=$ 1 - and high $C a-(b, c) C a=0.035$ and $\lambda=1 / 8$ and 1 respectively. At even higher $C a$ we observe the onset of a jet $-(d) C a=0.05, \lambda=1$. $(e, f) \log -\log$ plot of droplet volume versus capillary number. (e) $Q=1 / 4$, and $\square, \lambda=1 ; \bigcirc, 1 / 8$. (f) $\lambda=1 / 8 ; \square, Q=1 / 8 ; \bigcirc, 1 / 4 ; \mathbf{\square}$, $1 / 2$. At high $C a$, the droplet volume decreases more steeply, and is strongly influenced by the viscosity ratio $\lambda$.

blocks almost the entire cross-section of the main duct, and obstructs the flow of the continuous fluid. Regardless of the type of forcing, either fixed rate of flow or fixed pressure at the inlet of the continuous fluid, the obstruction of the main channel leads to an increase of pressure upstream of the tip of the dispersed fluid. We observe the details of the evolution of pressure in our numerical simulations.

As the dispersed-phase fluid enters the junction, the pressure rises gradually until the channel is blocked (figure $3 a, b$ ); this time interval is defined as the blocking time, $\tau_{\text {block. }}$. At this stage the continuous flow begins to squeeze the neck of the thread (figure $b, c$ ), until breakup occurs, and the pressure drops abruptly (figure $3 c, d$ ). The time interval from figure $3(b)$ to $3(d)$ is defined as squeezing time, $\tau_{\text {squeeze }}$. The viscosity 


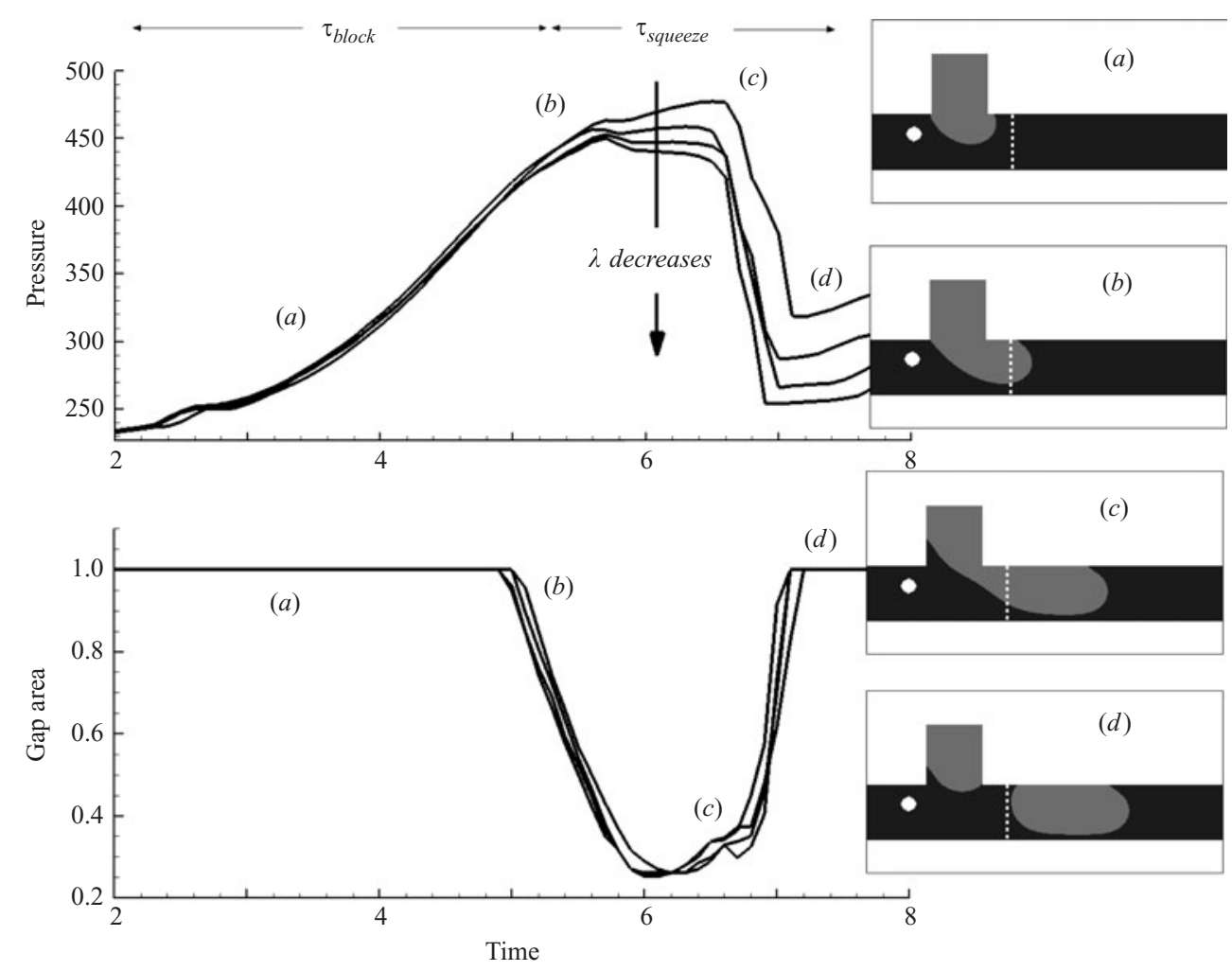

FIgURE 3. Pressure upstream of the T-junction (top) and area of the gap between the dispersed phase and the channel walls downstream (bottom) as a function of time; $C a=0.01$. The frames on the right-hand side visualize different stages of the droplet formation process. The pressure was measured at the position marked by the white dot, while the gap area is taken over a plane marked by the dashed line. We identify the two main steps of droplet formation: the interval $(a)-(b)$ mark the blocking phase, while from $(b)$ to $(d)$ the thread is squeezed until breakup occurs. Four curves are shown for each plot, with $\lambda=1,1 / 2,1 / 4$ and $1 / 8$. The pressure profile in the squeezing phase varies, somewhat weakly, with the viscosity ratio from the end of the blocking stage $(b)$ to the breakup point $(d)$.

contrast determines the details of the pressure profile in the squeezing phase, while its duration remains unaffected.

Also, we considered the behaviour of the pressure profile upstream of the junction for fixed $Q$ and $\lambda$, while varying the capillary number, $C a \ll 1$ (figure 4 ). We observe that $\tau_{\text {squeeze }}$ is independent of $\mathrm{Ca}$, while the blocking time grows as $\mathrm{Ca}$ is decreased. The results in figures 3 and 4 therefore confirm the intuitive fact that $\tau_{\text {squeeze }}$ depends only on the velocity of the outer fluid, which is responsible for the buildup of the pressure that is required to compress the neck, whose displacement will progress at a rate proportional to the flux of the dispersed phase.

When $Q_{d} \gg Q_{c}$ we expect $\tau_{\text {squeeze }} \gg \tau_{\text {block }}$ and the total time for droplet formation $\tau=\tau_{\text {block }}+\tau_{\text {squeeze }}$ depends only on $\tau_{\text {squeeze }}$, which suggests the relation $\tau \propto 1 / Q_{c}$. The volume of the droplet will therefore be $V_{d} \propto \tau Q_{d} \propto Q$. On the other hand, if $Q_{d} \ll Q_{c}$, $\tau \approx \tau_{\text {block }}$ depends on the distance the breakup point has to travel from the side inlet to enter the junction and on the flow rate $Q_{d}$. Thus, we expect $\tau=\tau_{\text {block }} \propto 1 / Q_{d}$, which implies $V_{d} \propto \tau Q_{d} \propto$ constant, which is independent of the flow ratio $Q$. The two regimes can be combined in the simple dimensionless relation $V=1+\alpha Q$, where 


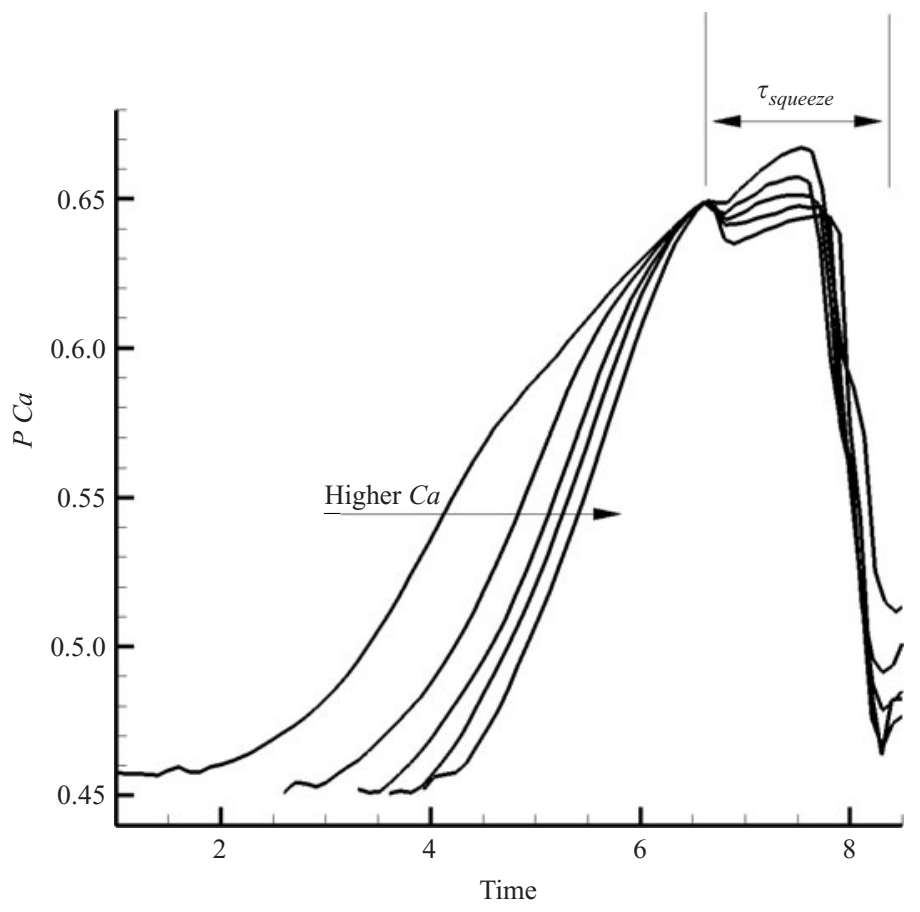

FiguRE 4. Time variation of the upstream pressure profile, scaled by the capillary number $(C a=0.002,0.004,0.006,0.008$ and 0.01 following the arrow from left to right). The time to achieve blocking decreases as the interfacial tension is decreased, while the squeezing time remains unchanged. $\lambda=1$ and $Q=1 / 4$.

$\alpha$ is a constant that may depend on the widths of the two channels. This mechanism was first proposed by Garstecki et al. (2006), where the functional form $V(Q)$ was observed experimentally. Cubaud et al. (2005) observed a similar behaviour for bubble formation in a symmetric cross-junction. We verified this result for the drop volume with our numerical simulations. Figure 5 shows the variation of the volume with $Q$ for three values of $\lambda$ and $C a=0.005$; the data collapse onto the same straight line $V=1+2 Q$.

\subsection{Transition from low to high $\mathrm{Ca}$}

As we increase the capillary number, while keeping the flow rates unchanged, we move away from the squeezing regime described in the previous section; the drop volume (figure 2) drops sharply with $C a$, and there is a strong dependence on the viscosity ratio. We observe that the incoming fluid only partially blocks the main channel (figure $2 b, c$ ), and that the breakup point moves downstream of the T-junction. This last effect is much more marked for $\lambda=1$. The overall trends are consistent with that observed for unbounded coflow, where the breakup point moves away from the inlet for higher viscosities of the dispersed phase (Zhang \& Stone 1997; Cramer et al. 2004). Also, the greater deformation of the more viscous thread is consistent with the stretch computed for two-dimensional droplets on a wall in a shear flow (Schleizer \& Bonnecaze 1999). On the other hand, in the unbounded case, the droplet size is approximately independent of the viscosity ratio, which is in clear contrast with the results of our simulations. The explanation for such a marked difference is related to 


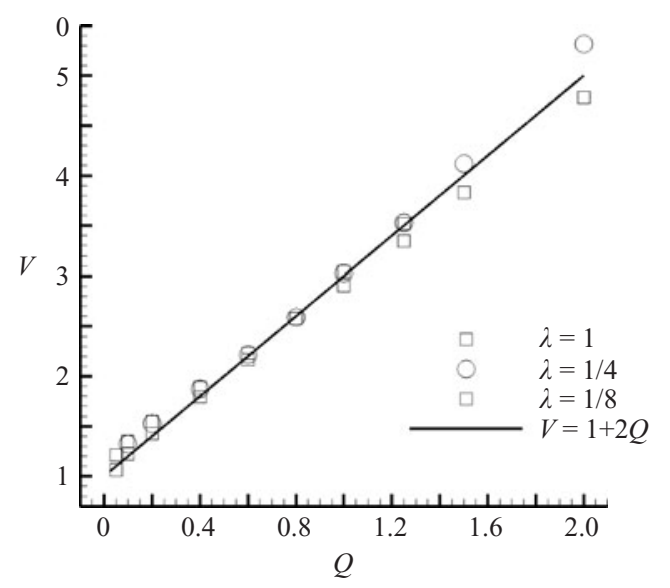

FIGURE 5. Variation of the dimensionless droplet volume with flow rate ratio for low capillary number, $\mathrm{Ca}=0.005$.

the constrained geometry: here, owing to the presence of the droplet, the changes in the flow of the outer fluid cannot be neglected.

We next contrast directly the pressure fluctuations, which accompany droplet formation, for the sequeezing regime $(C a \lesssim 0.01)$ and dripping regime $(C a \gtrsim 0.02)$; detailed results are given in figures 6 and 7 . We observe that for $\lambda=1$, the pressure fluctuations upstream of the T-junction decrease drastically as we move from the squeezing to the dripping regime. The perturbation of the outer flow owing to the presence of the droplet in the channel is significantly reduced, and the main driving force for the necking of the thread is the shear force. On the other hand, the pressure fluctuations maintain a much larger amplitude for $\lambda=1 / 8$ : droplet formation is still governed by the squeezing mechanism, while there might be some effect due to shear. Similar to the volume versus $\mathrm{Ca}$ plot of figure 2, we demonstrate the different qualitative nature of the squeezing and dripping regimes by considering the difference in the amplitude of the pressure fluctuations $\Delta p$ (figure 7) in the two regimes. Whereas for low $\mathrm{Ca}$ the amplitude remains constant, in the dripping regime there is a clear decrease, which is well pronounced for $\lambda=1$, and more gradual for $\lambda=1 / 8$. We believe that this behaviour, i.e. of a more gradual transition to dripping at lower values of $\lambda$, suggests that the description of the dynamics of breakup with the use of the capillary number calculated only for the continuous phase is not sufficient. For $\lambda$ significantly different from unity, we should take into account an effective capillary number calculated on the basis of capillary numbers for both phases.

\subsection{High Ca: the dripping regime}

In order to clarify the role of shear stresses in the dripping regime, we consider two model scaling relations proposed by Umbanhowar et al. (2000) for breakup in a coflowing unbounded fluid, and by Thorsen et al. (2001) for breakup in a T-junction. In both cases, we assume that breakup occurs when the interfacial force is nearly balanced by the shear force, such that $r \sim \sigma /(\mu \dot{\varepsilon})$ where $r$ is the final droplet radius and $\dot{\varepsilon}$ is the shear rate exerted on the droplet, based upon the mean speed of flow of the continuous phase. Thorsen et al. (2001) took into account that the emerging droplet decreases the cross-section of the channel available for the flow of the continuous fluid. In order to maintain the volumetric rate of flow, the continuous phase flows faster through the gap between the droplet and the wall of the channel, compared to the 


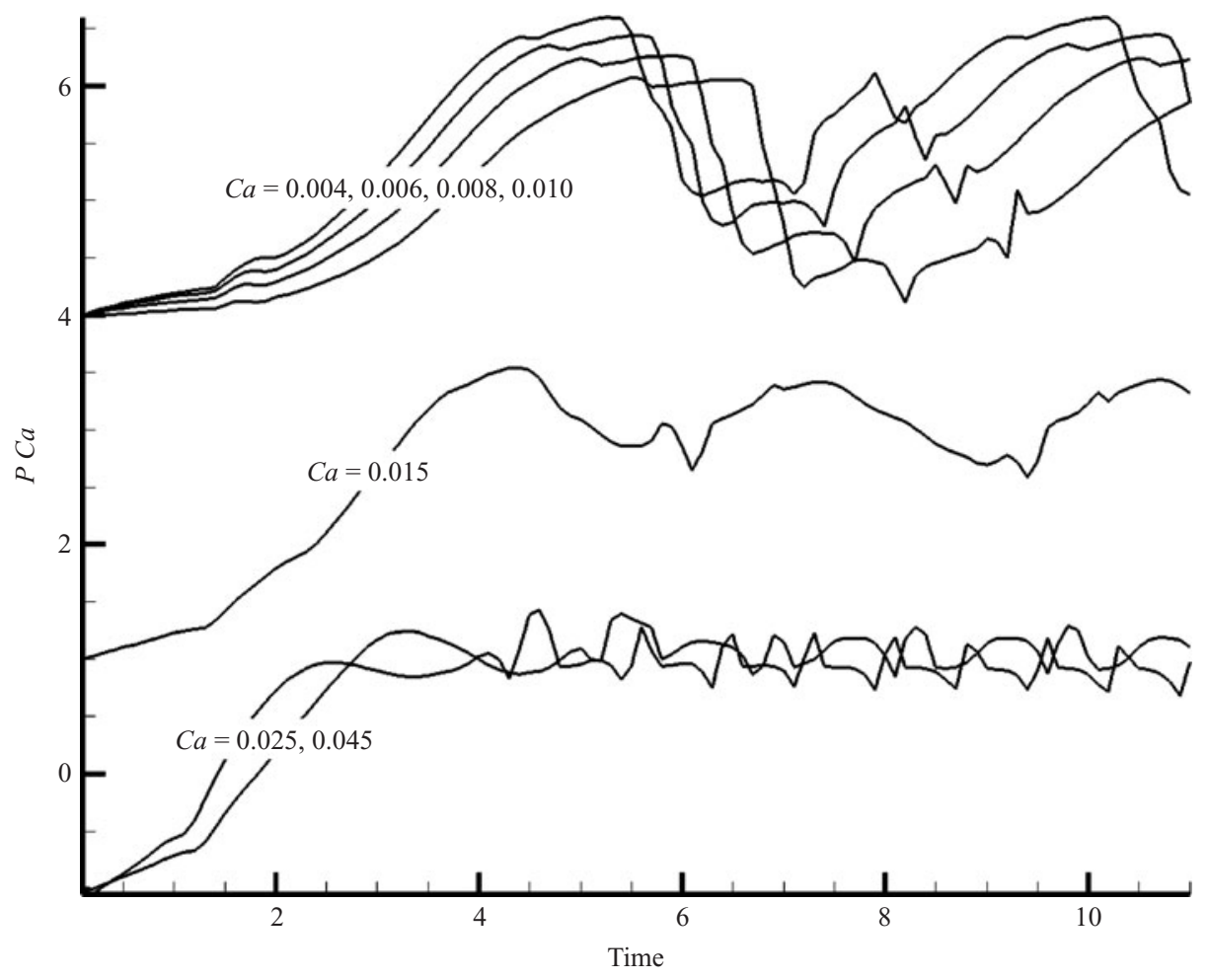

FiguRE 6. Pressure as a function of time upstream of the T-junction for different values of the capillary number. The oscillations correspond to the droplet formation frequency; as $\mathrm{Ca}$ is increased, the frequency increases while the amplitude of the pressure fluctuations decreases, as shown in the curves from top to bottom. The pressure on the vertical axis is rescaled by the capillary number, such that the amplitude of the fluctuations can be reported on the same scale for both the squeezing regime $(C a<0.015)$ and the dripping regime $(C a>0.015) . \lambda=1$ and $Q=1 / 4$. The sharp kinks appearing for the low capillary numbers are spurious effects due to the contact of droplets with the outlet in the simulation box.

case of an unobstructed channel, and exerts a larger shear stress on the droplet. This effect, in turn, results in the smaller size of the droplets that are formed in confined geometry - smaller than for the same superficial speed of flow of the continuous fluid in an unbounded case. In particular, Thorsen et al. (2001) calculated the shear stress on the basis of the measured size of the gap between the dispersed fluid and the sidewalls of the main channel. We performed a similar analysis: we measured the gap area in our simulations, and estimated $\dot{\varepsilon}$ as the ratio between the average flow speed in the gap, $v_{g}$, and linear width of the gap, $L_{g}$, estimated by the square root of the cross-section of the gap. We will use the dimensionless rescaled shear rate $\dot{\varepsilon}_{d}=\dot{\varepsilon} L / v_{c}$. Based on the dimensionless groups,

$$
R=\frac{r}{L} \sim \frac{1}{C a \dot{\varepsilon}_{d}} .
$$

In figure 8, we plot the radius of the droplet calculated on the basis of (4.1) and compare it to the radius measured in our simulations. The prediction overshoots the actual radius by an order of magnitude. In fact, in Thorsen et al. (2001), the calculated radii of the droplets were also systematically larger than the observed values. This discrepancy is consistent with the general observation that droplet breakup is much 


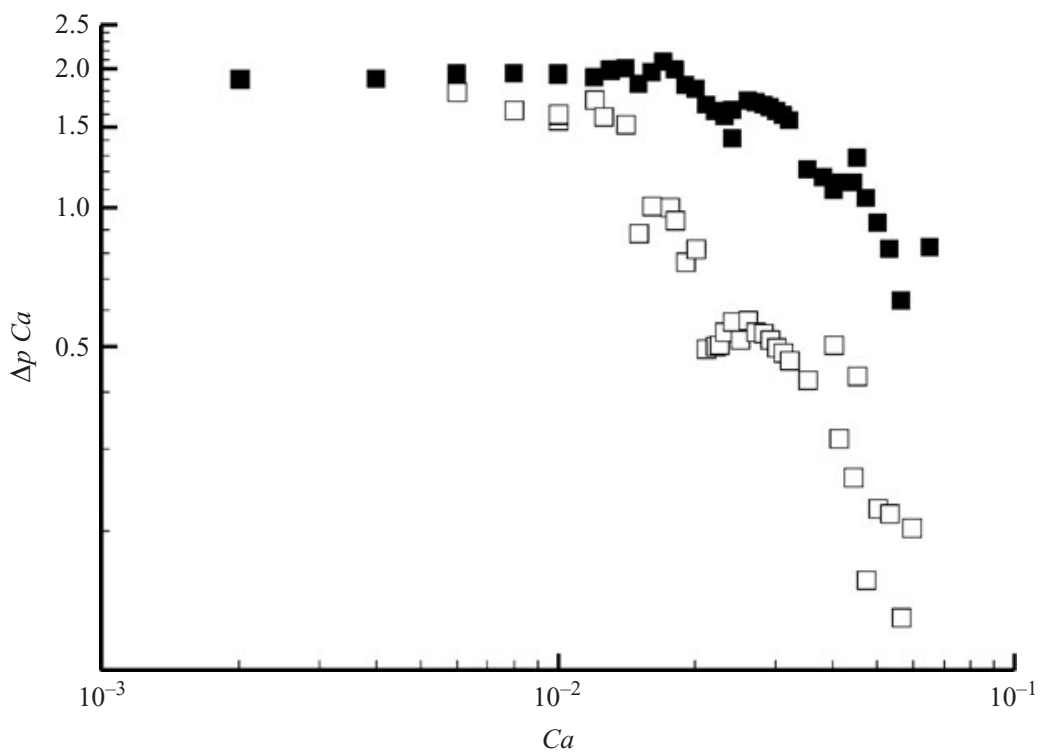

FIGURE 7. Variation of the pressure fluctuation $\Delta p$ upstream of the T-junction with the capillary number for two values of the viscosity ratio, $\square, \lambda=1$ and $\mathbf{\square}, \lambda=1 / 8$. The pressure is rescaled by the interfacial tension by multiplying by the capillary number $\mathrm{Ca}$. Similarly to figure 2, this diagram clearly illustrates the transition from the squeezing regime to the dripping regime, where there is a strong dependence on the viscosity ratio.

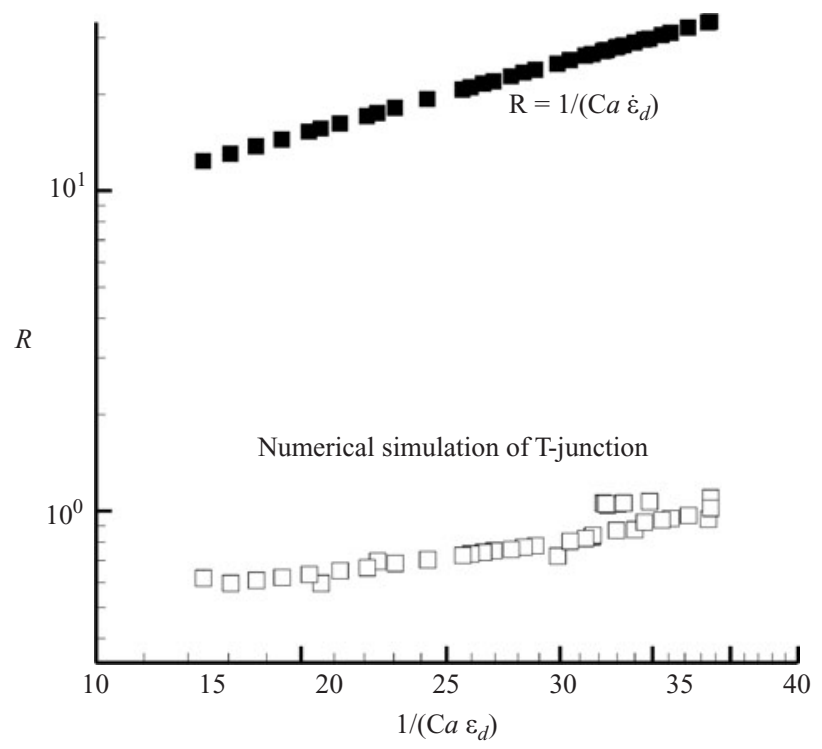

FIGURE 8. Variation of the droplet radius with the ratio between interfacial tension stresses and shear stresses, equal to $1 /\left(C a \dot{\varepsilon}_{d}\right)$, where $C a$ is the capillary number and $\dot{\varepsilon}_{d}$ is the dimensionless shear rate of the continuous phase. The data refer to the dripping regime $(C a>0.015) . Q=1 / 4$, $\lambda=1$. The open symbols are the results of the numerical simulations and the filled symbols correspond to the simple approximation, equation (4.1). 
easier in microfluidic devices than for unbounded flows. Even considering the actual shear flow in the breakup region, the critical capillary number can easily be ten times smaller than for free flow conditions (Link et al. 2004). From our simulations of the dripping regime, we find $R \approx 1 /\left(\mathrm{Ca} \dot{\varepsilon}_{d}\right)^{0.4}$, i.e. the radius of the droplet increases much more slowly than would be expected from the scaling law (4.1). A similar result was obtained by van van der Graaf et al. (2006) for an even less confined system - a T-junction with a wider main channel - for which the authors reported the linear size of the droplets to scale with the capillary number as $\mathrm{Ca}^{-0.25}$. All the observations above, point to the fact that there are additional forces exerted on the droplet that pull or push it downstream and speed up the breakup. Since we observe that even in the dripping regime the droplets typically occupy a substantial portion of the cross-section of the microchannel, we can associate this additional force with an elevated pressure drop along the length of the emerging droplet, which is the same effect that played the dominant role in the squeezing regime.

Summarizing, although the process of formation of droplets in the dripping regime is reminiscent of dripping in coflow in an unbounded fluid, it is fundamentally different because the pressure effects are never negligible, unless the radius of the droplet is much smaller than the width of the channel. The combination of shear stresses and the pressure makes quantitative analysis of the scaling of the size of the droplets formed in this regime particularly difficult (note that the range of the values of capillary number over which we observe dripping is quite small). As the capillary number is further increased, the breakup point moves progressively downstream from cycle to cycle, and a jet is formed. There clearly is a transition from the stable dripping regime to a jetting regime. We were not, however, able to explore this regime in detail, as it requires very large simulation boxes.

\section{Discussion}

Our numerical study of the dynamics of breakup in a T-junction has revealed three distinct regimes of formation of droplets: squeezing, dripping and jetting. The first regime - squeezing - is particular to microfluidic geometries because it depends crucially on strong physical confinement of the interfacial dynamics. The evolution of pressure that we observe in our simulations upstream of the emerging droplet confirms the model of breakup proposed in Garstecki et al. (2006) and Guillot \& Colin (2005). In this regime, the dominating contribution in the dynamics of breakup arises from the buildup of pressure, which results from the blocking of the cross-section of the main channel by the tip of the dispersed phase. We observe this regime at low values of capillary numbers with the transition to a regime in which the shear stresses exerted on the emerging droplet begin to play a significant role at $C a \sim 0.015$ for $\lambda=1$, and at slightly higher values for $\lambda=1 / 8$. The size (volume) of the droplets and bubbles formed in the squeezing regime can be described by a simple scaling relation of the form: $V=1+\alpha Q$, where $\alpha$ is a constant of order unity, whose exact value depends on the geometry of the T-junction, and is independent of $\lambda$ (Garstecki et al. 2006). There are two important characteristics of the squeezing regime: (i) the lack of dependence of the size of the droplets (bubbles) on material parameters (e.g. viscosities of the fluids or interfacial tension) make this mode of breakup particularly simple for use in applications, allowing for varying the fluids without altering the dynamics of formation of the discrete elements of fluid, and (ii) the fact that breakup in this regime is not driven by shear stresses exerted on the emerging droplets (bubbles) allows for 
emulsification in conditions in which the capillary number can be very small - that is even in very small devices and at very small rates of flow.

We observe that the dripping, or shear-driven, breakup regime, is strongly modified in the microfluidic setting of a T-junction geometry with respect to the analogous mode of breakup in unbounded fluids, owing to the effects of confinement. Even when the shear stresses exerted on the emerging droplet are able to deform it substantially, it still occupies a significant portion of the cross-section of the channel. Thus, the effects of buildup of pressure upstream of the emerging droplet are still present, and they modify the condition for breakup (facilitating it). Owing to a small range of the values of the capillary number over which we observe dripping - before it is replaced by jetting - it is not reasonable to propose any scaling relation for the size of the droplets formed in this regime. In the jetting regime, the stream of the dispersed fluid extends downstream of the T-junction and the two immiscible fluids are in laminar flow side-by-side over lengths of the channel which are at least several times larger than its width.

M.D.M. acknowledges support from Unilever Corporate Research and the European Union (MRTN-CT-2004-005728). H. A.S. thanks the Harvard MRSEC (DMR-0213805) and Unilever Research for support. P. G. thanks the Foundation for Polish Science for financial support.

\section{REFERENCES}

Anderson, D. M., McFadden, G. B. \& Wheeler, A. A. 1998 Diffuse-interface methods in fluid mechanics. Аnnu. Rev. Fluid Mech. 30, 139-165.

Anna, S. L., Bontoux, N. \& Stone, H. A. 2003 Formation of dispersions using flow focusing in microchannels. Appl. Phys. Lett. 82, 364-366.

Badalassi, V. E., Ceniceros, H. D. \& Banerjee, S. 2003 Computation of multiphase systems with phase field models. J. Comput. Phys. 190, 371.

BASARAN, O. A. 2002 Small-scale free surface flows with breakup: drop formation and emerging applications. AIChE J. 48, 1842-1848.

Batchelor, G. K. 1967 An Introduction to Fluid Mechanics. Cambridge University Press.

Blackmore, B., Li, D. Q. \& GaO, J. 2001 Detachment of bubbles in slit microchannels by shearing flow. J. Colloid Interface Sci. 241, 514-520.

CAHn, J. W. 1965 Phase separation by spinodal decomposition in isotropic systems. J. Chem. Phys. 42, 93-99.

CAHN, J. W. 1977 Critical point wetting. J. Chem. Phys. 66, 3667-3672.

Christopher, G. F. \& ANNA, S. L. 2007 Microfluidic methods for generating continuous droplet streams. J. Phys. D: Appl. Phys. 40, R319-R336.

Cornish, V. W. 2006 Catalytic competition for cells. Nature 440, 156-157.

Cramer, C., Fischer, P. \& Windhab, E. J. 2004 Drop formation in a co-flowing ambient fluid. Chem Engng Sci. 59, 3045-3058.

Cristini, V., Balwzdziewicz, J. \& Loewenberg, M. 1998 Drop breakup in three-dimensional viscous flows. Phys. Fluids 10, 1781-1783.

Cristini, V. \& TAN, Y.-C. 2004 Theory and numerical simulation of droplet dynamics in complex flows - a review. Lab Chip 4, 257-264.

Cubaud, T. \& Ho, C. M. 2004 Transport of bubbles in square microchannels. Phys. Fluids 16, 4575-4585.

Cubaud, T., Tatieni, M. T., Zhong, X. \& Ho, C. M. 2005 Bubble dispenser in microfluidic devices. Phys. Rev. E 172, 037302.

Cygan, Z. T., Cabral, J. T., Beers, K. L. \& Amis, E. J. 2005 Microfluidic platform for the generation of organic-phase microreactors. Langmuir 21, 3629-3634.

De Menech, M. 2006 Modeling of droplet breakup in a microfluidic T-shaped junction. Phys. Rev. E 73, 031505. 
Dendukuri, D., Tsoi, K., Hatton, T. A. \& Doyle, P. S. 2005 Controlled synthesis of nonspherical microparticles using microfluidics. Langmuir 21, 2113-2116.

Dreyfus, R., Tabeling, P. \& Willaime, H. 2003 Ordered and disordered patterns in two-phase flows in microchannels. Phys. Rev. Lett. 90, 144505.

Duffy, D. C., McDonald, J. C., Schueller, O. J. A. \& Whitesides, G. M. 1998 Rapid prototyping of microfluidic systems in poly(dimethylsiloxane). Ann. Chem. 70, 4974-4984.

Dupuis, A. \& Yeomans, J. M. 2004 Lattice Boltzmann modelling of droplets on chemically heterogeneous surfaces. Future Generation Comput. Syst. 20, 993-1001.

Engl, W., Roche, M., Colin, A., Panizza, P. \& Ajdari, A. 2005 Droplet traffic at a simple junction at low capillary numbers. Phys. Rev. Lett. 95, 208305.

Feng, J. Q. \& Basaran, O. A. 1994 Shear flow over a translationally-symmetric cylindrical bubble pinned on a slot in a plane wall. J. Fluid Mech. 275, 351-378.

Ganán-Calvo, A. M. \& Gordillo, J. M. 2001 Perfectly monodisperse microbubbling by capillary flow focusing. Phys. Rev. Lett. 87, 274501.

Garstecki, P., Gitlin, I., Diluzio, W., Whitesides, G., Kumacheva, E. \& Stone, H. A. 2004 Formation of monodisperse bubbles in a microfluidic flow-focusing device. Appl. Phys. Lett. 85, 2649-2651.

Garstecki, P., Fuerstman, M. \& Whitesides, G. M. 2005a Design for mixing using bubbles in branched microfluidic channels. Appl. Phys. Lett. 86, 244108.

Garstecki, P., Fuerstman, M. \& Whitesides, G. M. $2005 b$ Nonlinear dynamics of a microfluidic flow-focusing bubble generator. Phys. Rev. Lett. 94, 234502.

Garstecki, P., Fuerstman, M. \& Whitesides, G. M. 2005c Oscillations with uniquely long periods in a microfluidic bubble generator. Nature Phys. 1, 168-171.

Garstecki, P., Stone, H. A. \& Whitesides, G. M. 2005d Mechanisms for flow-rate controlled breakup in confined geometries: a route to monodisperse emulsions. Phys. Rev. Lett. 94, 164501.

Garstecki, P., Feuerstman, M., Stone, H. A. \& Whitesides, G. M. 2006 Formation of droplets and bubbles in a microfluidic T-junction: scaling and mechanism of breakup. Lab Chip 6, 437-446.

Gerdts, C. J., Sharoyan, D. E. \& Ismagilov, R. F. 2004 A synthetic reaction network: chemical amplification using nonequilibrium autocatalytic reactions coupled in time. J. Am. Chem. Soc. 126, 6327-6331.

van der Graaf, S., Nisisako, T., Schroen, C. G. P. H., van der Sman, R. G. M. \& Boom, R. M. 2006 Lattice Boltzmann simulations of droplet formation in a T-shaped microchannel. Langmuir 22, 4144-4152.

Guillot, P. \& Colin, A. 2005 Stability of parallel flows in a microchannel after a T junction. Phys. Rev. E 72, 066301.

Gunther, A. \& Jensen, K. F. 2006 Multiphase microfluidics: from flow characteristics to chemical and materials synthesis. Lab Chip 6, 1487-1503.

Gunther, A., Kahn, S. A., Thalmann, M., Trachsel, F. \& Jensen, K. F. 2004 Transport and reaction in microscale segmented gas-liquid. Lab Chip 4, 278-286.

He, M. Y., Edgar, J. S., Jeffries, G. D. M., Lorenz, R. M., Shelby, J. P. \& ChiU, D. T. 2005 Selective encapsulation of single cells and subcellular organelles into picoliter-and femtoliter-volume droplets. Ann. Chem. 77, 1539-1544.

JACQMIN, D. 1999 Calculation of two-phase Navier-Stokes flows using phase-field modelling. J. Comput. Phys. 155, 96-127.

JACQMin, D. 2000 Contact-line dynamics of a diffuse fluid interface. J. Fluid Mech. 402, 57-88.

Jensen, M. J., Stone, H. A. \& Bruus, H. 2006 A numerical study of two-phase Stokes flow in an axisymmetric flow-focusing device. Phys. Fluids 18, 077103.

Jeong, W., Kim, J. Y., Kim, S., Lee, S., Mensing, G. \& Beebe, D. J. 2004 Hydrodynamic microfabrication via 'on the fly' photopolymerization of microscale fibers and tubes. Lab Chip 4, 576-580.

Jeong, W. J., Kim, J. Y., Choo, J., Lee, E. K., Han, C. S., Beebe, D. J., Seong, G. H. \& Lee, S. H. 2005 Continuous fabrication of biocatalyst immobilized microparticles using photopolymerization and immiscible liquids in microfluidic systems. Langmuir 21, 3738-3741.

Kenis, P. J. A., Ismagilov, R. F. \& Whitesides, G. M. 1999 Microfabrication inside capillaries using multiphase laminar flow patterning. Science 285, 83-85. 
KIM, J. 2005 A continuous surface tension force formulation for diffuse-interface models. J. Comput. Phys. 204, 784-804.

Kunsenok, O., Jasnow, D., Yeomans, J. \& Balazs, A. C. 2003 Periodic droplet formation in chemically patterned microchannels. Phys. Rev. Lett. 91, 108303.

Li, M., Zheng, L. \& Harris, M. T. 2000 Modeling the synthesis of porous spherical shells and microspheres of zirconia by electrodispersion precipitation. J. Powder Technol. 110, $15-21$.

Li, X. \& Pozrikidis, C. 1996 Shear flow over a liquid drop adhering to a solid surface. J. Fluid Mech. 307, 167-190.

Link, D. R., Anna, S. L., Weitz, D. A. \& Stone, H. A. 2004 Geometrically mediated breakup of drops in microfluidic devices. Phys. Rev. Lett. 92, 053403.

McDonald, J. C., Duffy, D. C., Anderson, J. R., Chiu, D. T., Wu, H., Schueller, O. J. A. \& Whitesides, G. M. 2000 Fabrication of microfluidic systems in poly(dimethylsiloxane). Electrophoresis 21-40, 27.

Nisisako, T., ToriI, T. \& Higuchi, T. 2004 Novel microreactors for functional polymer beads. Chem. Engng J. 101, 23-29.

Okushima, S., Nisisako, T., ToRiI, T. \& Higuchi, T. 2004 Controlled production of monodisperse double emulsions by two-step breakup in microfluidic devices. Langmuir 20, 9905-9908.

Osher, S. \& FedKiw, R. P. 2001 Level set methods: an overview and some recent results. J. Comput. Phys. 169, 463-502.

Pedersen, H. \& Horvath, C. 1981 Axial dispersion in a segmented gas-liquid flow. Ind. Engng Chem. Fund. 20, 181-186.

Rallison, J. M. 1984 The deformation of small viscous drops and bubbles in viscous flow. Annu. Rev. Fluid. Mech. 16, 45-66.

SCARDOVELli, R. \& ZALESKI, S. 1999 Direct numerical simulation of free-surface and interfacial flows. Annu. Rev. Fluid Mech 31, 567-603.

Schleizer, A. D. \& Bonnecaze, R. T. 1999 Displacement of a two-dimensional immiscible droplet adhering to a wall in shear and pressure-driven flows. J. Fluid Mech. 383, 29-54.

Sia, S. K., Linder, V., Parviz, B. A., Siegel, A. \& Whitesides, G. M. 2004 An integrated approach to a portable low-cost immunoassay for resource-poor settings. Angew. Chem. Intl Edn 43, 498-502.

Song, H. \& Ismagilov, R. F. 2003 Millisecond kinetics on a microfluidic chip in multiphase microfluidics at low values of the Reynolds and capillary numbers. J. Am. Chem. Soc. 125 , $14613-14619$.

Song, H., Bringer, M. R., Tice, J. D., Gerdts, C. J. \& Ismagilov, R. F. 2003 Experimental test of scaling of mixing by chaotic advection moving through microfluidic channels. Appl. Phys. Lett. 83, 4664-4666.

Song, H., Chen, D. L. \& Ismagilov, R. F. 2006 Reactions in droplets in microfluidic channels. Angew. Chem. Intl Edn 45, 7336-7356.

SQuires, T. \& Quake, S. 2005 Microfluidics: fluid physics at the nanoliter scale. Rev. Mod. Phys. 77, 977-1026.

Stone, H. A. 1994 Dynamics of drop deformation and breakup in viscous fluids. Annu. Rev. Fluid. Mech. 26, 65-102.

Stone, H. A. 2005 On lubrication flows in geometries with zero curvature. Chem. Engng Sci. 60, 4838-4845.

Stone, H. A., Stroock, A. D. \& Ajdari, A. 2004 Engineering flows in small devices: Microfluidics toward a lab-on-a-chip. Annu. Rev. Fluid. Mech 36, 381-411.

Subramaniam, A. B., AbKarian, M. \& Stone, H. A. 2005 Controlled assembly of jammed colloidal shells on fluid droplets. Nature Materials 4, 553-556.

Sugiura, S., Nakajima, M., Iwamoto, S. \& Seki, M. 2001 Interfacial tension driven monodisperse droplet formation from microfabricated channel array. Langmuir 17, 5562-5566.

Sugiura, S., Oda, T., Izumida, Y., Aoyagi, Y., Satake, M., Ochial, A. \& Ohкohchi, N. 2005 Size control of calcium alginate beads containing living cells using micro-nozzle array. Biomaterials 26, 3327-3331.

Suryo, R. \& Basaran, O. A. 2006 Tip streaming from a liquid drop forming from a tube in a co-flowing outer fluid. Phys. Fluids 18, 082102. 
Takeuchi, S., Garstecki, P., Weibel, D. B. \& Whitesides, G. M. 2005 An axisymmetric flowfocusing microfluidic device. Adv. Materials 17, 1067-1072.

TAYLOR, G. I. 1934 The formation of emulsions in definable fields of flow. Proc. R. Soc. Lond. A 146, 501-523.

Thorsen, T., Roberts, R. W., Arnold, F. H. \& Quake, S. R. 2001 Dynamic pattern formation in a vesicle-generating microfluidic device. Phys. Rev. Lett. 86, 4163-4166.

Tice, J. D., Lyon, A. D. \& Ismagilov, R. F. 2004 Effects of viscosity on droplet formation and mixing in microfluidic channels. An. Chim. Acta 507, 73-77.

Umbanhowar, P. B., Prasad, V. \& Weitz, D. A. 2000 Monodisperse emulsion generation via drop break off in a coflowing stream. Langmuir 16, 347-351.

Utada, A. S., Lorenceau, E., Link, D. R., Kaplan, P. D, Stone, H. A. \& Weitz, D. A. 2005 Monodisperse double emulsions generated from a microcapillary device. Science 308, 537 541.

Utada, A. S., Fernandez-Nieves, A., Stone, H. A. \& Weitz, D. A. 2007 Dripping to jetting transitions in co-flowing liquid streams. Phys. Rev. Lett. 99, 094502.

WaGner, A. J. \& Yeomans, Y. M. 1997 Effect of shear on droplets in a binary mixture. Intl J. Mod. Phys. C 8, 773-782.

Wagner, A. J., Wilson, L. M. \& Cates, M. E. 2003 Role of inertia in two-dimensional deformation and breakdown of a droplet. Phys. Rev. E 68, 045301(R).

Ward, T., Faivre, M., Abkarian, M. \& Stone, H. A. 2005 Microfluidic flow focusing: drop size and scaling in pressure versus flow-rate-driven pumping. Electrophoresis 26, 37163724.

Wilkes, E. D., Phillips, S. \& Basaran, O. 1999 Computational and experimental analysis of dynamics of drop formation. Phys. Fluids 11, 3577-3598.

XU, Q. \& NaKaJima, M. 2004 The generation of highly monodisperse droplets through the breakup of hydrodynamically focused microthread in a microfluidic device. Appl. Phys. Lett. 85, 3726-3728.

Xu, S. Q., Nie, Z. H., Seo, M., Lewis, P., Kumacheva, E., Stone, H. A., Garstecki, P., Weibel, D. B., Gitlin, I. \& Whitesides, G. M. 2005 Generation of monodisperse particles by using microfluidics: control over size, shape, and composition. Angew. Chem. Intl Edn 44, 724-728.

Zhang, D. F. \& Stone, H. A. 1997 Drop formation in viscous flows at a vertical capillary tube. Phys. Fluids 9, 2234-2242.

Zheng, B. \& Ismagilov, R. F. 2005 A microfluidic approach for screening submicroliter volumes against multiple reagents by using preformed arrays of nanoliter plugs in a three-phase liquid/liquid/gas flow. Angew. Chem. Intl Edn 44, 2520-2523.

Zheng, B., RoAch, L. S. \& Ismagilov, R. F. 2003 Screening of protein crystallization conditions on a microfluidic chip using nanoliter-size droplets. J. Am. Chem. Soc. 125, 11 170-11 171.

Zhou, C., Yue, P. \& Feng, J. J. 2006 Formation of simple and compound drops in microfluidic devices. Phys. Fluids 18, 092105. 